\title{
Dos procedimientos de ajuste por riesgo para la estadía hospitalaria como indicador de desempeño
}

\author{
Two risk adjustment methods for length of stay as indicator of \\ hospital performance
}

\author{
DrC. Teddy Osmin Tamargo Barbeito, DraC. Rosa Eugenia Jiménez \\ Paneque, Dra. Ángela Rosa Gutiérrez Rojas, Dra. I sabel Mora Díaz
}

Hospital Clinicoquirúrgico "Hermanos Ameijeiras. La Habana, Cuba.

\section{RESUMEN}

Introducción: la estadía hospitalaria, es el indicador por excelencia de la eficiencia de los servicios prestados, cualquier valoración sobre el desempeño hospitalario que se realice a partir de este indicador debe contemplar de alguna manera las características de los pacientes que se utilizaron para estimar su valor.

Objetivo: evaluar dos procedimientos de ajuste por riesgo para la estadía hospitalaria como indicador de desempeño.

Métodos: se realizó un estudio retrospectivo en el servicio de medicina interna del hospital "Hermanos Ameijeiras" entre mayo y octubre de 2006. La muestra de 606 historias clínicas, se dividió aleatoriamente en dos partes: 304 historias clínicas para la estimación de la estadía esperada a partir de la función de regresión lineal múltiple y de la clasificación de pacientes por Grupos de Diagnósticos Relacionados, y otro de 302 historias clínicas para la validación de los dos métodos de ajuste por riesgo. En la fase de validación, se evaluó la capacidad de cada procedimiento para detectar ineficiencias de la atención en otro grupo de historias clínicas, por medio del análisis de varianza y la construcción de una curva ROC.

Resultados: influyeron en la estadía hospitalaria, la edad, el índice de gravedad, el diagnóstico principal al egreso y sus interacciones, y el sexo. El área bajo la curva ROC con la regresión lineal múltiple fue de 0,747 ( $p<0,001)$ (IC de $95 \%$ 0,690$0,805)$ y con los Grupos de Diagnósticos Relacionados, fue de $0,738(p<0,001)$ (IC de $95 \%$ 0,680-0,796). 
Conclusiones: ambos procedimientos de ajuste son igualmente eficaces en la detección de problemas de eficiencia, pero el modelo de regresión lineal múltiple, para estimar la estadía esperada, resulta mejor que el de los Grupos de Diagnósticos Relacionados por razones principalmente económicas. Este hecho avala su utilización en países o instituciones de recursos limitados como los propios de países no desarrollados.

Palabras clave: Estadía hospitalaria, eficiencia, Grupos de Diagnósticos Relacionados, ajuste por riesgo.

\section{ABSTRACT}

Introduction: the length of stay at hospital is the indicator of the rendered service efficiency par excellence, so any assessment of the hospital performance based on this indicator should take into account in one way or another the characteristics of the patients considered for the value estimation.

Objective: to evaluate two risk adjustment methods for the length of stay as indicator of the hospital performance.

Methods: a retrospective study was conducted at the internal medicine service of "Hermanos Ameijeiras" hospital from May to October, 2006. The sample of 606 medical histories was randomly divided into two parts, that is, one group comprised 304 medical histories for the predicted length of stay estimation from the multiple linear regression function and from the patient classification by related diagnosis groups, and the other included 302 medical histories for the validation of these two risk adjustment methods. In the validation phase, the capacity of each procedure to detect care deficiencies in another group of medical histories through the variance analysis and the ROC curve construction was evaluated.

Results: age, severity index, main diagnosis on discharge and its interactions, and sex influenced the length of stay. The area under the ROC curve with the multiple linear regression was $0.747(p<0,001)(95 \% \mathrm{Cl}, 0.690-0.805)$ whereas the same are with the related diagnosis groups was $0,738(p<0,001)(95 \% \mathrm{Cl}, 0.680-$ $0.796)$.

Conclusions: both risk adjustment methods are equally effective in detecting efficiency problems, but the multiple linear regression model is better than the related diagnosis groups in estimating the predicted length of stay due to economic reasons. This aspect supports its use in poor-resource countries or institutions as is the case of underdeveloped countries.

Key words: Length of stay at hospital, efficiency, Related Diagnosis Groups, risk adjustment. 


\section{NTRODUCCIÓN}

La estadía o estancia hospitalaria, es el indicador por excelencia del aprovechamiento de la cama y por tanto de la eficiencia de los servicios prestados, ${ }^{1,2}$ pero es bien conocido que sus cambios no solo están supeditados a problemas en la eficiencia ${ }^{3}$ sino también a las características de los pacientes, ${ }^{2,4}$ que constituyen la materia prima para su cálculo.

Se necesita por tanto distinguir en cada estadía qué parte de la misma se debe a las características del paciente y qué parte a la eficiencia y calidad del servicio prestado. Al procedimiento de contemplar los factores que influyen sobre determinado evento que se va a utilizar como indicador (mortalidad, complicaciones, estadía) se le denomina "ajuste por riesgo" y a los indicadores que se obtienen de ese ajuste, "indicadores ajustados."1,5

Entre los factores identificados por su asociación con la estadía, independiente de la calidad de la atención, se encuentran la edad, ${ }^{2,6}$ el diagnóstico, la gravedad de la enfermedad, el sexo, las enfermedades asociadas, ${ }^{2}$ el tipo de tratamiento, la raza, la etnia, el estado civil, el tipo de admisión (urgente o electivo), ${ }^{4}$ algunas características psicosociales del paciente, ${ }^{3,7}$ la fuente de remisión de los pacientes, el tipo de sistema de pago de la atención médica y el tipo de especialidad del médico. ${ }^{8}$

La gravedad de la enfermedad es una variable que ha sido muy utilizada por los investigadores para el ajuste por riesgo, a través del tiempo se han desarrollado una serie de índices tanto generales ${ }^{9,10} \operatorname{como}^{\text {específicos }}{ }^{11}$ y se han empleado también con el mismo propósito los llamados sistemas de clasificación de pacientes, ${ }^{12-14}$ además de otras medidas indirectas de la gravedad como las enfermedades asociadas. ${ }^{15,16}$

Los Grupos de Diagnósticos Relacionados (GDR) conforman el sistema de clasificación de pacientes más universalmente utilizado para el ajuste por riesgo, pero no han sido pocas las críticas que se le han hecho. Librero y otros, ${ }^{13}$ mencionan que el ajuste a partir de los GDR no está exento de problemas conceptuales y metodológicos y que no son tan homogéneos internamente como se piensa. No existen antecedentes del uso de los GDR en Cuba probablemente por lo costoso de su adquisición.

Por otro lado, el ajuste por riesgo puede hacerse directamente con datos recogidos de la historia clínica del paciente, sin necesidad de utilizar sistemas comerciales ya que el conocimiento sobre los factores que influyen sobre cualquier resultado de la atención médica, en particular sobre la estadía, es amplio y basta con emplear modelos estadísticos como la regresión múltiple o el análisis de varianza y covarianza para hacer comparaciones ajustadas. La dificultad estriba entonces en la factibilidad de emplear tales modelos y recoger la información necesaria para el ajuste.

En Cuba, en el hospital "Hermanos Ameijeiras", se realizó un estudio donde se desarrolla y valida un índice de gravedad para servicios clínicos llamado índice de Gravedad de Servicios Clínicos del Hospital "Hermanos Ameijeiras" (IGCHHA), ${ }^{10}$ el cual ha sido utilizado en el ajuste de la estadía hospitalaria para detectar ineficiencias en los servicios hospitalarios. ${ }^{2}$

En esencia, lo que se propone es un indicador de eficiencia basado en la diferencia entre la estadía observada y la esperada. La estadía esperada se estima de acuerdo a determinadas características de los pacientes, entre las que se encuentra la 
gravedad, medida a partir de los índices desarrollados en el mismo contexto mediante una función de regresión lineal múltiple (RLM). La diferencia existente entre una y otra sería un indicio de ineficiencia del servicio hospitalario brindado. Las variables para la función son las que señala la literatura y los coeficientes en la función de regresión se estiman en el propio medio hospitalario.

Teniendo en cuenta las desventajas que tiene la clasificación según GDR, el presente trabajo se propone evaluar dos procedimientos de ajuste por riesgo para la estadía hospitalaria como indicador de desempeño.

\section{MÉTODOS}

Se realizó un estudio retrospectivo en el servicio de medicina interna del hospital "Hermanos Ameijeiras" durante los meses de mayo a octubre de 2006, donde se compararon dos métodos de ajuste por riesgo con respecto a su capacidad para la detección de ineficiencias de la atención hospitalaria.

\section{Universo y muestra}

Se revisaron las 714 historias clínicas de los pacientes que egresaron vivos del servicio de medicina interna en el período y se eliminaron 70 historias que no se encontraban en el archivo, 10 que pertenecían a pacientes extranjeros, 7 que no contaban con todos los datos necesarios y 21 que pertenecían a pacientes incluidos ya por otro egreso en el mismo período. De modo que la muestra quedó conformada por un total de 606 historias clínicas.

Las 606 historias clínicas que entraron en el estudio se dividieron aleatoriamente en 2 partes: 304 historias clínicas para la estimación de la estadía esperada por medio de la función de regresión lineal múltiple y la clasificación por GDR y otro de 302 historias clínicas para la validación de los dos métodos de ajuste por riesgo. De este último grupo se eliminaron 25 historias por no contar con un valor de la estadía mediana según GDR estimada en el primer grupo, pues ese GDR no estaba presente en el grupo de la estimación, lo que hace un total de 277 historias clínicas efectivas en el segundo grupo.

\section{Análisis estadístico}

En la fase de estimación o de entrenamiento, se emplearon dos procedimientos para estimar la estadía esperada para cada paciente: 1. Mediante la estimación de una función de regresión lineal múltiple utilizando como variable dependiente la estadía hospitalaria y las explicativas: la edad, el sexo, el índice de gravedad, el reingreso, la estancia en unidad de cuidados intensivos $(\mathrm{UCl})$, las enfermedades asociadas y el diagnóstico principal al egreso. Se consideraron también las interacciones de todas las variables de diseño o dummy variables* correspondientes al diagnóstico principal al egreso con el índice de gravedad y 2. Mediante el empleo de las medianas de los GDR que se encuentran en el grupo de estimación.

La variable estadía hospitalaria fue transformada con el logaritmo de base diez por la conocida asimetría derecha que tiene esta variable. Se comprobó el logro de la 
normalidad después de hacer la transformación mediante el histograma de la variable y las pruebas de normalidad.

Para la selección del modelo óptimo se eliminaron los valores aberrantes (residuos estandarizados mayores de tres desviaciones estándar); lo que se realizó dos veces. Se utilizó el método paso a paso, conocido como "Wald hacia delante" (Forward Wald), para obtener la función final.

Se realizó además análisis de la multicolinealidad que incluyó obtención del factor de inflación de la varianza (FIV), la tolerancia $\left(1-R^{2}\right)$, el índice de condición y los autovalores. Para todas las variables el valor del FIV fue menor de 10, las tolerancias se encontraban todas por encima de 0,1 ; todos los índices de condición fueron menores que 15 y la mayoría de los autovalores fueron diferentes de 0 . No se consideró entonces la exclusión de ninguna variable por esta razón.

Se identificaron todos los coeficientes $\left(b_{i}\right)$ que fueran diferentes de 0 de manera significativa a nivel poblacional $(p<0,05)$, mediante el estadígrafo t de student.

En la fase de validación se buscó la existencia de problemas de la eficiencia de la atención clasificados como eficiencia adecuada, problemas moderados de eficiencia en la atención y problemas graves y muy graves de eficiencia en la atención.

Se calculó para cada paciente la estadía esperada de dos maneras: 1. Mediante la función estimada con el primer grupo de historias y 2. La esperada mediante la clasificación por GDR.

Después se procedió a calcular la diferencia entre la estadía observada y la esperada calculada estimada por los dos procedimientos. Se evaluó entonces la relación entre estas diferencias y la variable eficiencia de la atención. Para ello se calcularon la media y desviación estándar de la diferencias para cada categoría de esta variable y mediante el análisis de varianza de clasificación simple (ANOVA) con la prueba de Scheffé a posteriori, se compararon los tres grupos de la clasificación de la eficiencia de la atención.

Por último, para evaluar la capacidad de los dos métodos en la detección de problemas de eficiencia de la atención, se hizo el cálculo del área bajo la curva con su intervalo de confianza de $95 \% .{ }^{17}$ La variable eficiencia de la atención, dejando dos categorías: atención adecuada e inadecuada (problemas moderados, graves y muy graves de la atención).

Para evaluar la diferencia en la capacidad de detección de cada procedimiento, para encontrar problemas de eficiencia en la atención, se compararon ambas áreas bajo la curva ROC mediante el estadígrafo ji cuadrado de homogeneidad de las áreas bajo las curvas, utilizando el programa estadístico Epidat 3.1.

En todas las pruebas de hipótesis se fijó un nivel de significación $\alpha=0,05$.

\section{RESULTADOS}

El promedio de la edad y del índice de gravedad en el grupo empleado en la estimación de la función fue ligeramente menor que el del grupo de la validación. La mediana de la estadía hospitalaria para el grupo de la estimación fue algo mayor 
que en el de la validación. No existieron diferencias estadísticamente significativas entre los dos grupos (tabla 1 ).

El diagnóstico principal más frecuente al egreso, en ambos grupos, fue el seis (tumores benignos, enfermedades endocrinometabólicas, de la piel y del tejido conectivo, del sistema osteomioarticular, infecciosas y parasitarias y las anemias). El sexo predominante en ambos grupos fue el femenino, las enfermedades asociadas fueron mayores en el grupo empleado en la estimación. El reingreso se comportó de manera similar en ambos grupos. Existió diferencia significativa en el caso de la estancia en la $\mathrm{UCl}$. La distribución de las variables en ambos grupos fue homogénea (tabla 1 ).

Tabla 1. Características de los grupos incluidos

\begin{tabular}{|c|c|c|c|c|c|c|}
\hline \multirow{2}{*}{\multicolumn{2}{|c|}{ Variables cuantitativas }} & \multicolumn{2}{|c|}{$\begin{array}{l}\text { Grupo estimación } \\
N=304\end{array}$} & \multicolumn{2}{|c|}{$\begin{array}{c}\text { Grupo validación } \\
N=277\end{array}$} & \multirow[t]{2}{*}{$p$} \\
\hline & & Media & $\mathrm{DE}^{2}$ & Media & $\mathrm{DE}^{\mathrm{a}}$ & \\
\hline \multicolumn{2}{|l|}{ Edad } & 58,5 & 17,7 & 60,3 & 17,5 & 0,183 \\
\hline \multicolumn{2}{|c|}{ Índice de gravedad } & 84,3 & 27,4 & 86,7 & 25,3 & 0,156 \\
\hline \multicolumn{2}{|l|}{ Estadía } & $\begin{array}{c}10,0 \\
(10,7)\end{array}$ & 7,0 & $\begin{array}{c}9,8 \\
(8,0)\end{array}$ & 6,0 & 0,088 \\
\hline \multicolumn{2}{|c|}{ Variables cualitativas } & No. & $\%$ & No. & $\%$ & \\
\hline \multirow{7}{*}{$\begin{array}{l}\text { Diagnóstico } \\
\text { principal } \\
\text { al egreso }\end{array}$} & Diagnóstico 1 & 42 & 13,8 & 34 & 12,3 & \multirow{7}{*}{0,112} \\
\hline & Diagnóstico 2 & 57 & 18,8 & 40 & 14,4 & \\
\hline & Diagnóstico 3 & 53 & 17,4 & 68 & 24,5 & \\
\hline & Diagnóstico 4 & 29 & 9,5 & 18 & 6,5 & \\
\hline & Diagnóstico 5 & 10 & 3,3 & 8 & 2,9 & \\
\hline & Diagnóstico 6 & 80 & 26,3 & 88 & 31,8 & \\
\hline & Diagnóstico 7 & 33 & 10,9 & 21 & 7,6 & \\
\hline \multirow{2}{*}{ Sexo } & Masculino & 109 & 35,9 & 121 & 43,7 & \multirow{2}{*}{$0,066^{\mathrm{e}}$} \\
\hline & Femenino & 195 & 64,1 & 156 & 56,3 & \\
\hline \multirow{2}{*}{$\begin{array}{l}\text { Enfermedades } \\
\text { asociadas }\end{array}$} & Sí & 154 & 50,7 & 125 & 45,1 & \multirow{2}{*}{$0,211^{\mathrm{e}}$} \\
\hline & No & 150 & 49,3 & 152 & 54,9 & \\
\hline \multirow{2}{*}{ Reingresos } & Sí & 4 & 1,3 & 4 & 1,4 & \multirow{2}{*}{1,000} \\
\hline & No & 300 & 98,7 & 273 & 98,6 & \\
\hline \multirow{2}{*}{ Estancia en UCI } & Sí & 11 & 3,6 & 13 & 4,7 & \multirow{2}{*}{0,002} \\
\hline & No & 293 & 96,4 & 264 & 95,3 & \\
\hline
\end{tabular}

a Desviación estándar, "Mediana, "Prueba t de Student, "Prueba ji cuadrado sin corrección de Yates, "Prueba ji cuadrado con corrección de Yates, 'Test de probabilidades exactas de Fisher. Diagnóstico 1: Respiratorio, Diagnóstico 2: Cardiovascular, Diagnóstico 3: Digestivo, Diagnóstico 4: T, Malignos, Diagnóstico 5: Genitourinario, Diagnóstico 6: T, benignos, enf endoc metab, de la piel y TCSC, tejido conectivo, del SOMA, anemias e infecciosas y parasitarias, Diagnóstico 7: Otros

Las variables incluidas en el modelo final fueron la edad, el IGCHHA, el diagnóstico principal al egreso representada por el grupo diagnóstico tres (enfermedades digestivas), las interacciones de los grupos diagnóstico dos (enfermedades cardiovasculares) y tres (enfermedades digestivas) con el índice de gravedad y el sexo (tabla 2).

Con el procedimiento basado en la regresión lineal múltiple, los pacientes con atención adecuada tuvieron, en promedio, aproximadamente dos días menos de estadía hospitalaria observada que la esperada, sin embargo para los de problemas graves y muy graves la estadía observada fue superior a la esperada en cinco días. Existieron diferencias significativas entre los tres valores de medias $(p<0,001)$. Las

http://scielo.sld.cu 
diferencias fueron entre todos los posibles pares de medias que se forman entre las tres categorías de la clasificación de la variable eficiencia de la atención (Fig. 1).

Tabla 2. Resultados de la regresión lineal múltiple

\begin{tabular}{|c|c|c|c|c|c|}
\hline \multirow{2}{*}{ Variable } & \multirow{2}{*}{ Coef. (b) } & \multirow{2}{*}{$\mathrm{EE}^{\mathrm{a}}$} & \multicolumn{2}{|c|}{ IC $95 \%$} & \multirow{2}{*}{$p$} \\
\hline & & & LI & LS & \\
\hline Constante & 0,811 & 0,054 & 0,704 & 0,918 & $p<0,001$ \\
\hline Edad & $-0,004$ & 0,001 & $-0,006$ & $-0,002$ & $p<0,001$ \\
\hline ÍGCHHA & 0,005 & 0,001 & 0,003 & 0,006 & $p<0,001$ \\
\hline Diagnósticos & \multicolumn{5}{|l|}{ Dummy } \\
\hline Diagnóstico 1 & \multicolumn{5}{|c|}{ Categoría de referencia (respiratorio) } \\
\hline Diagnóstico 2 & 0,027 & 0,166 & $-0,300$ & 0,354 & 0,958 \\
\hline Diagnóstico 3 & $-0,689$ & 0,109 & $-0,904$ & $-0,474$ & $p<0,001$ \\
\hline Diagnóstico 4 & 0,326 & 0,302 & $-0,268$ & 0,921 & 0,860 \\
\hline Diagnóstico 5 & $-0,198$ & 0,356 & $-0,897$ & 0,502 & 0,935 \\
\hline Diagnóstico 6 & $-0,004$ & 0,151 & $-0,301$ & 0,293 & 0,977 \\
\hline Diagnóstico 7 & 0,146 & 0,165 & $-0,178$ & 0,470 & 0,940 \\
\hline $\mathrm{D} 2 *$ IGCHHA & $-0,001$ & 0,000 & $-0,002$ & 0,000 & $p<0,001$ \\
\hline D3* IGCHHA & 0,007 & 0,001 & 0,004 & 0,010 & 0,005 \\
\hline $\operatorname{Sexo}^{b}$ & 0,061 & 0,027 & 0,008 & 0,113 & 0,023 \\
\hline Resumen modelo & \multicolumn{5}{|c|}{$R^{2}=0,336, R^{2}$ corregido $=0,322$} \\
\hline
\end{tabular}

"Error estándar, ${ }^{\circ}$ Categoría de referencia sexo masculino,

Diagnóstico 1: Respiratorio, Diagnóstico 2: Cardiovascular, Diagnóstico 3: Digestivo, Diagnóstico 4: $T$, Malignos, Diagnóstico 5: Genitourinario, Diagnóstico 6: T, benignos, enf, endoc, metab, de la piel y TCSC, tejido conectivo, del SOMA, anemias e infecciosas y parasitarias, Diagnóstico 7: Otros

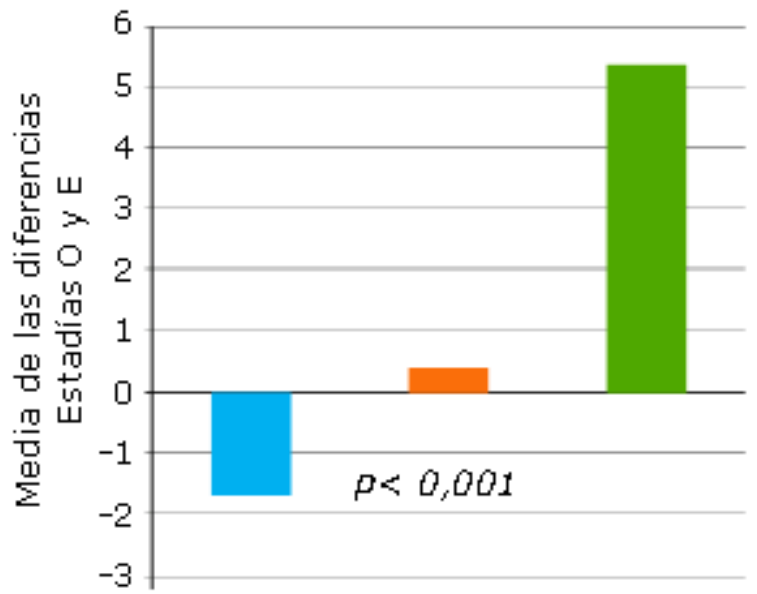

Adecuada

Problem as moderados de eficiencia

Problemas graves y muy graves de eficiencia

Fig. 1. Promedio del indicador estadía observada (EO) menos esperada (E), estimado mediante la RLM, según eficiencia de la atención médica. 
El área bajo la curva ROC que indica la capacidad para la detección de las ineficiencias moderadas a muy graves de la atención, del indicador estadía observada menos esperada estimado por el modelo de regresión lineal múltiple, fue de $0,747(p<0,001)$ (IC de $95 \% 0,690-0,805)$ (Fig. 2 ).

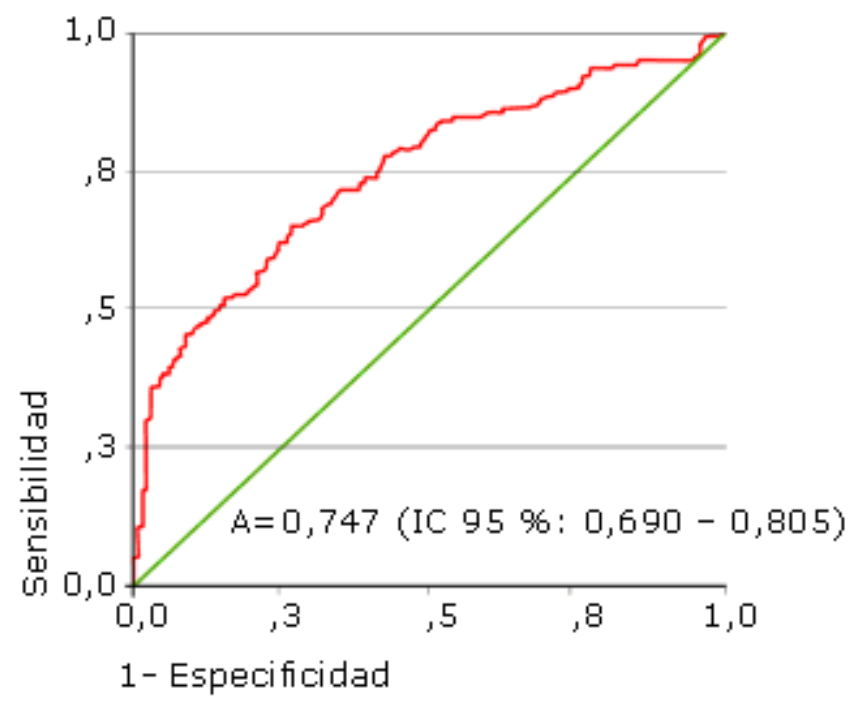

Fig. 2. Curva ROC para el indicador estadía observada menos esperada estimado mediante la RLM.

En la tabla 3 se puede comprobar que para el punto de corte - 1 día se obtuvieron los mejores valores de sensibilidad y especificidad 75,2 \% y 67,9 \%, respectivamente. El punto de corte -3 días mostró una sensibilidad de $87,2 \%$ en buena medida alta, y el punto de corte 2 días tuvo una especificidad de $88,1 \%$ que puede considerarse también elevada.

Con el procedimiento que utiliza el sistema de clasificación por GDR, los pacientes con atención adecuada tuvieron en promedio aproximadamente 2 días menos de estadía hospitalaria esperada con respecto a la observada y los que tuvieron ineficiencias graves y muy graves permanecieron en el hospital ingresados aproximadamente 6 días más que lo esperado. Existieron diferencias significativas entre los tres valores de medias $(p<0,001)$. Las diferencias fueron entre la atención adecuada y los problemas graves o muy graves de la atención, así como entre estos y los problemas moderados de eficiencia de la atención (Fig. 3). 
Tabla 3. Sensibilidad, especificidad y valores predictivos para diferentes puntos de corte en la diferencia estadía observada-estadía esperada estimada por medio de la función de regresión múltiple

\begin{tabular}{|c|c|c|c|c|}
\hline $\begin{array}{l}\text { Punto } \\
\text { de corte }\end{array}$ & Sensibilidad & Especificidad & $\begin{array}{c}\text { Valores } \\
\text { predictivos } \\
\text { positivos* }\end{array}$ & $\begin{array}{c}\text { Valores } \\
\text { predictivos } \\
\text { negativos* }\end{array}$ \\
\hline-9 & 1,000 & 0,037 & 0,507 & 1,000 \\
\hline-7 & 0,992 & 0,060 & 0,513 & 1,000 \\
\hline-5 & 0,940 & 0,172 & 0,545 & 1,000 \\
\hline-4 & 0,925 & 0,306 & 0,588 & 0,804 \\
\hline-3 & 0,872 & 0,440 & 0,639 & 0,776 \\
\hline-2 & 0,827 & 0,552 & 0,689 & 0,763 \\
\hline-1 & 0,752 & 0,679 & 0,756 & 0,734 \\
\hline 0 & 0,647 & 0,739 & 0,792 & 0,678 \\
\hline 1 & 0,564 & 0,821 & 0,847 & 0,655 \\
\hline 2 & 0,496 & 0,881 & 0,893 & 0,638 \\
\hline 3 & 0,391 & 0,993 & 0,993 & 0,622 \\
\hline 5 & 0,271 & 0,955 & 0,957 & 0,569 \\
\hline 9 & 0,150 & 0,985 & 0,985 & 0,539 \\
\hline 13 & 0,075 & 0,993 & 0,993 & 0,502 \\
\hline 17 & 0,053 & 1,000 & 1,000 & 0,516 \\
\hline
\end{tabular}

* Prevalencia (Probabilidad a priori de tener ineficiencias de la atención)= 0,498.

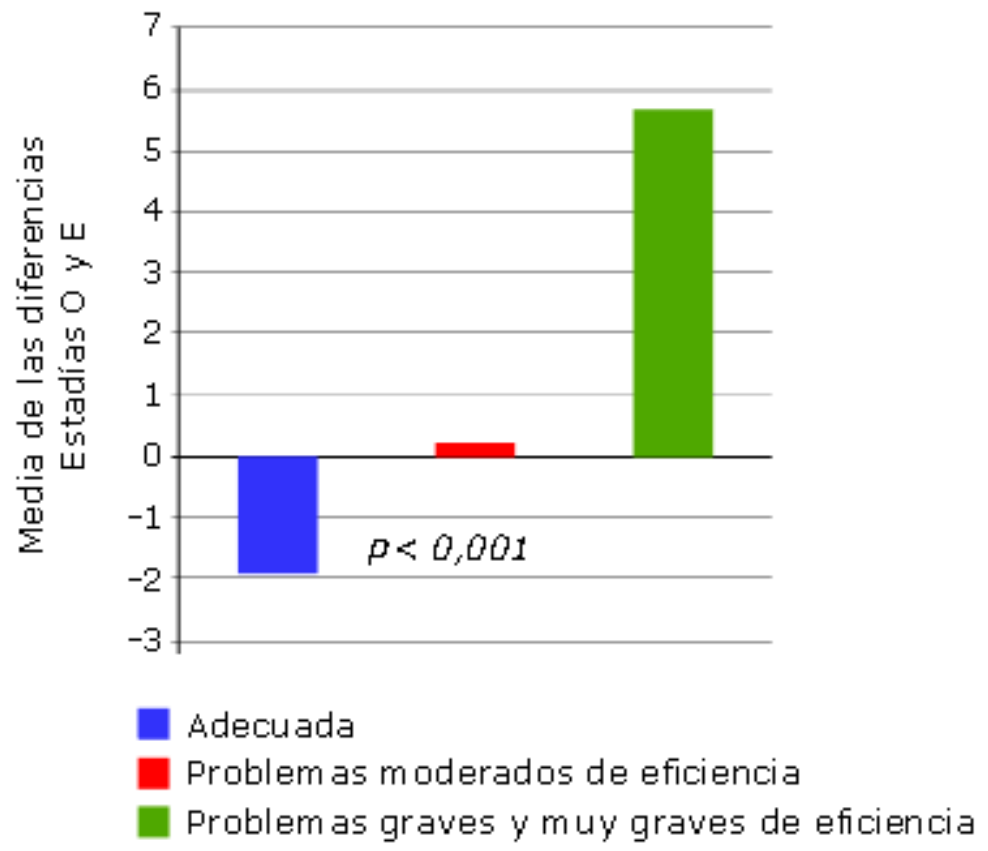

Fig. 3. Promedio del indicador estadía observada (EO) menos esperada (E), estimado mediante los GDR, según eficiencia de la atención médica. 
El área bajo la curva ROC que indica la capacidad para la detección de las ineficiencias moderadas a muy graves de la atención, del indicador estadía observada menos esperada estimado por la clasificación por GDR, fue de 0,738 ( $p<$ 0,001) (IC de $95 \%$ 0,680-0,796) (Fig. 4).

Se puede observar que para el punto de corte 0 día se obtuvieron los mejores valores de sensibilidad y especificidad con cifras de $61,7 \%$ y $75,4 \%$, respectivamente. El punto de corte -3 días ofreció un valor de sensibilidad de 89,5 $\%$ razonablemente alto, y el punto de corte 2 días tuvo una especificidad de $85,8 \%$ que también puede considerarse alta (tabla 4).

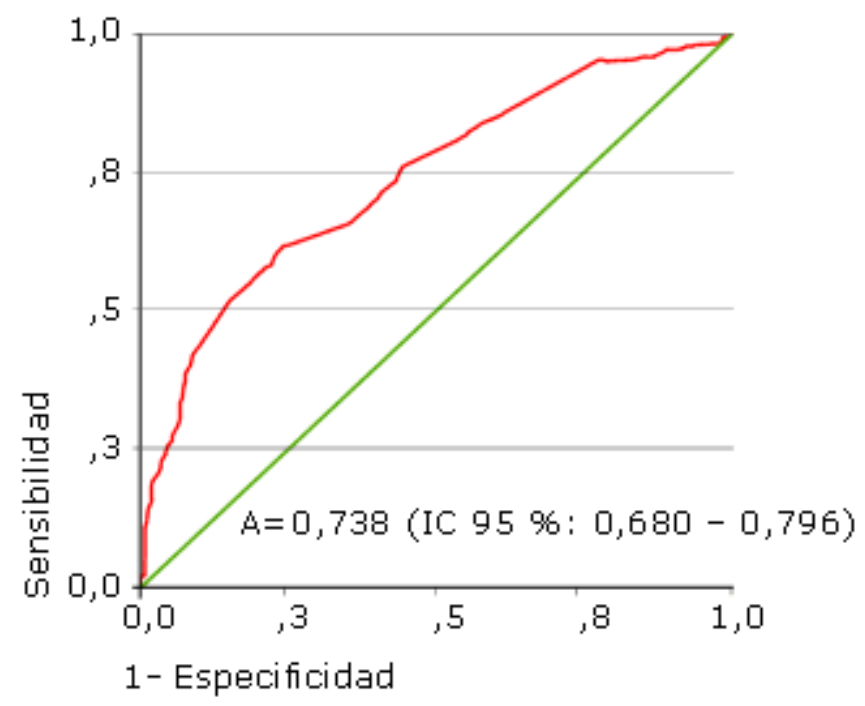

Fig. 4. Curva ROC para el indicador estadía observada menos esperada estim ado mediante la clasificación por GDR.

No existieron diferencias significativas entre las dos áreas bajo las curvas ROC $(p=0,7168)$ por lo que los dos procedimientos parecen ser igualmente eficaces y útiles para la detección de ineficiencias moderadas a muy graves de la atención (tabla 5, Fig. 5). 
Tabla 4. Sensibilidad, especificidad y valores predictivos para diferentes puntos de corte en la diferencia estadía observada-estadía esperada estimada por medio de la clasificación por GDR

\begin{tabular}{|c|c|c|c|c|}
\hline Punto de corte & Sensibilidad & Especificidad & $\begin{array}{c}\text { Valores } \\
\text { predictivos } \\
\text { positivos* }\end{array}$ & $\begin{array}{c}\text { Valores } \\
\text { predictivos } \\
\text { negativos* }\end{array}$ \\
\hline-14 & 1,000 & 0,007 & 0,500 & 1,000 \\
\hline-10 & 0,985 & 0,037 & 0,507 & 0,713 \\
\hline-6 & 0,962 & 0,142 & 0,536 & 0,790 \\
\hline-5 & 0,955 & 0,216 & 0,559 & 0,829 \\
\hline-4 & 0,940 & 0,261 & 0,573 & 0,814 \\
\hline-3 & 0,895 & 0,328 & 0,596 & 0,759 \\
\hline-2 & 0,805 & 0,470 & 0,652 & 0,708 \\
\hline-1 & 0,722 & 0,567 & 0,696 & 0,673 \\
\hline 0 & 0,617 & 0,754 & 0,801 & 0,665 \\
\hline 1 & 0,534 & 0,813 & 0,841 & 0,638 \\
\hline 2 & 0,481 & 0,858 & 0,875 & 0,625 \\
\hline 4 & 0,323 & 0,925 & 0,930 & 0,579 \\
\hline 7 & 0,195 & 0,963 & 0,964 & 0,547 \\
\hline 10 & 0,150 & 0,970 & 0,971 & 0,535 \\
\hline 16 & 0,060 & 0,993 & 0,993 & 0,516 \\
\hline 27 & 0,023 & 1,000 & 1,000 & 0,508 \\
\hline
\end{tabular}

* Prevalencia (Probabilidad a priori de tener ineficiencias de la atención) = 0,498.

Tabla 5. Comparación de las dos áreas bajo las curvas

\begin{tabular}{|l|c|c|c|c|}
\hline $\begin{array}{l}\text { Procedimiento de } \\
\text { estimación }\end{array}$ & $\begin{array}{c}\text { Áreas bajo } \\
\text { la curva }\end{array}$ & $\begin{array}{c}\text { Estadígrafo ji } \\
\text { cuadrado }\end{array}$ & $\begin{array}{c}\text { Grados de } \\
\text { libertad }\end{array}$ & Probabilidad \\
\hline RLM & 0,747 & 0,1316 & 1 & 0,7168 \\
\hline GDR & 0,738 & 0.96 & 1 \\
\hline
\end{tabular}




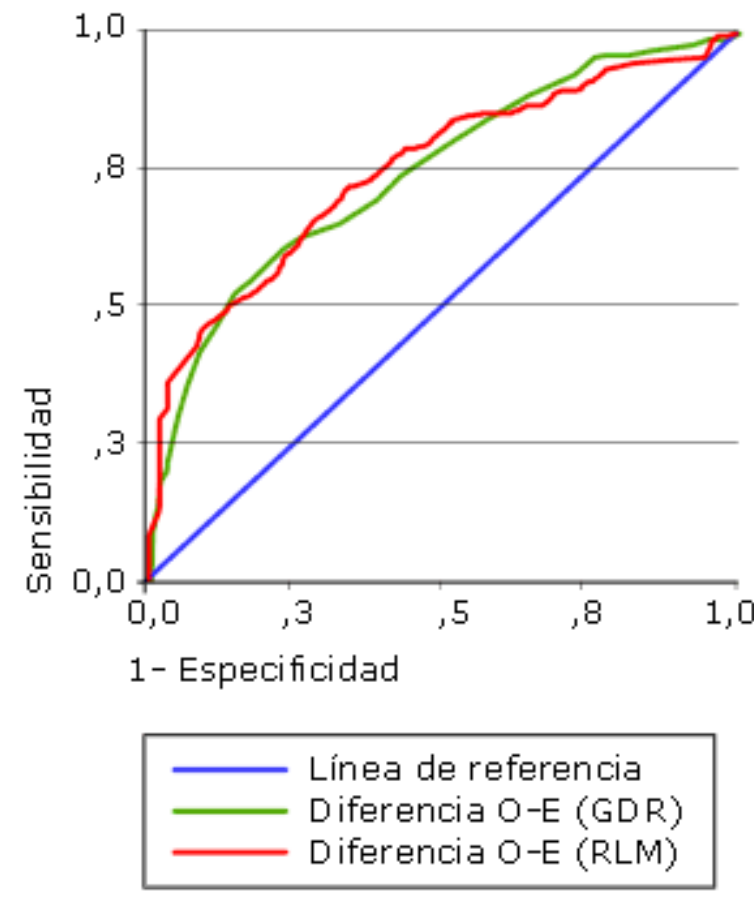

Fig. 5. Curva ROC para el indicador estadía observada (OE) menos esperada, estimado mediante la clasificación por GDR.

\section{DISCUSIÓN}

La estadía hospitalaria en los servicios de medicina interna parece ser similar en distintas latitudes, por ejemplo Becchi y otros, ${ }^{18}$ en un estudio donde se evalúan las características de un grupo de pacientes adultos hospitalizados en un servicio de medicina interna, registran una estadía mediana de 9 días, algo similar a la de esta investigación.

Con respecto a las características de los pacientes incluidos en el estudio, también se encuentran semejanzas con los incluidos en otras investigaciones realizadas en regiones diferentes. Por ejemplo, Everett y otros, ${ }^{19}$ comparan los costos y la estadía de tres modelos de atención a pacientes en servicios de medicina interna y con edades promedio de 66,$5 ; 62,8$ y 58,6 años.

El diagnóstico principal al egreso fue clasificado de manera similar en un estudio anterior realizado en el hospital. ${ }^{2}$ No se encontraron otras referencias de una clasificación semejante del diagnóstico principal al egreso aunque es frecuente la alusión al diagnóstico y su influencia sobre la estadía hospitalaria. Prácticamente, no es posible, en un trabajo que abarca a todos los pacientes de un servicio de medicina interna, incluir enfermedades por separado y cada investigador utiliza la clasificación más acorde a sus fines.

El predominio del sexo femenino es notable en este estudio pero este hecho no se repite en muchas investigaciones. Everett y otros, ${ }^{19}$ en tres tipos de servicios de medicina interna observan una distribución del sexo femenino superior al masculino por lo que coinciden con esta investigación, sin embargo Horwitz y otros, ${ }^{20}$ en un servicio de medicina interna docente encuentran un predominio del sexo masculino y en uno no docente del femenino. 
Las variables independientes en estudio solo explican el $34 \%$ de la variación total de la estadía pero, según parece, es difícil encontrar un ajuste mejor a los datos cuando se trabaja con la estadía hospitalaria, probablemente por la cantidad de factores disímiles que pueden influir sobre esta variable. En un estudio anterior realizado en el hospital "Hermanos Ameijeiras", Jiménez y otros, ${ }^{2}$ obtienen un valor del coeficiente de determinación múltiple $\left(R^{2}\right)$ de 0,41 en el servicio de medicina interna. Otros autores, ${ }^{19}$ también en un servicio de medicina interna, obtienen un valor de 0,39 . Sahadevan, ${ }^{21}$ en una muestra de pacientes geriátricos procedentes de un servicio de medicina general y geriatría comunican el resultado de tres funciones para ajustar la estadía con valores de $\left(R^{2}\right)$ de $0,08,0,29$ y 0,33.

Las áreas bajo la curva ROC encontradas para el indicador en ambos casos, tienen valores que se pueden considerar en un rango aceptable (ambas mayores de 0,7 ) según la valoración que hacen Gursel y Demirtas sobre este aspecto. ${ }^{22}$ Hartz y otros, ${ }^{23}$ empleando tres métodos para la evaluación de la eficiencia de la atención a pacientes ingresados por neumonía encuentran áreas bajo la curva ROC de 0,72, 0,67 y 0,84 .

Con la regresión múltiple, el mejor punto de corte (mejor balance entre sensibilidad y especificidad) para detectar ineficiencias, implica que la estadía observada sea un día menor que la esperada mientras que con los GDR el mejor punto de corte es el que implica que ambas estadías sean iguales. Este es sin dudas un resultado sorprendente pues la idea inicial es que una estadía observada mayor que la esperada podría ser causada por ineficiencias en la atención. Sin embargo, la comparación univariada señala alrededor de siete días más de estadía promedio en el grupo de pacientes en los que se detectaron problemas graves de eficiencia con respecto al de eficiencia adecuada en ambos métodos de ajuste. Si se reconoce que lo más importante en la práctica es confirmar la presencia de ineficiencias, es decir, una alta especificidad, puntos de corte de tres días o más serían más adecuados y a su vez se acompañan de Valores Predictivos Positivos (VPP) muy altos con esta prevalencia de ineficiencias en la atención. Para la gestión hospitalaria un falso positivo tendría consecuencias peores que un falso negativo.

La idea de utilizar la diferencia entre estadía observada y esperada como procedimiento para realizar ajuste por riesgo, o para otros, contemplar el llamado case-mix que permita evaluar la eficiencia de la atención y comparar en espacio y tiempo no es nueva. Bernard y y Ryan, ${ }^{24,25}$ proponen un indicador basado en esta diferencia con iguales propósitos.

Los métodos que estiman la estadía esperada a base de modelos de regresión, tienen la ventaja de ser de fácil adaptación a condiciones locales y de fácil actualización y son de muy bajo costo puesto que programas de computación que realicen los cálculos pertinentes son, hoy día, asequibles a cualquier centro o servicio de salud. El modelo de regresión lineal múltiple puede ser reajustado con periodicidad incluyendo nuevos aspectos que se surjan de la práctica médica.

Los resultados obtenidos apoyan la hipótesis de que ambos métodos de ajuste son igualmente efectivos en la detección de problemas de eficiencia, una parte importante de la calidad de la atención. Se comprueba también que, con un método de aplicación relativamente simple en el contexto cubano actual, podrían obtenerse evaluaciones informadas de la eficiencia con que se están prestando los servicios hospitalarios, una cualidad imprescindible para cualquier sistema de salud público como el de Cuba y en particular en condiciones de país en desarrollo.

No obstante, algunas limitaciones del estudio pueden señalarse. La primera y más importante quizás es que otros tipos de problemas de calidad debidos a la atención 
médica propiamente dicha, no fueron abordados. La decisión sobre el tiempo de las demoras que podían considerarse ineficientes, se basó en el sentido común y la práctica en el hospital y no en una evaluación teórica sobre el problema pues se establecieron los intervalos de tiempo que se consideraron como normales para el buen funcionamiento de todos los servicios del hospital. La estimación de la función para estimar la estadía esperada se realiza en el mismo sitio donde se supone que existen las ineficiencias pues no se cuenta con un servicio de estadía ideal para este fin. Esta es la práctica seguida en otros estudios similares ya mencionados pero no hay dudas de que puede constituir una limitación. La eliminación de los valores atípicos (outliers) durante la búsqueda del modelo tiene como objetivo minimizar esta limitación. Otra limitación importante fue la escasez de bibliografía para la comparación y explicación de los resultados obtenidos, pues en la actualidad existe una tendencia a realizar estudios similares para enfermedades específicas y no de forma general en servicios de medicina interna. El escaso tamaño de muestra dentro de algunos GDR constituyó otra dificultad para la estimación de la estadía esperada.

Se concluye que la diferencia "estadía observada-estadía esperada" tiene una buena capacidad para la detección de las ineficiencias en la atención hospitalaria, cuando la estadía esperada contempla las características individuales de los pacientes y brinda una valiosa información para la toma de decisiones en el servicio de medicina interna.

El modelo de regresión lineal múltiple, para estimar la estadía esperada, resulta mejor que el de los GDR por razones principalmente económicas. Este hecho avala su utilización en países o instituciones de recursos limitados como los propios de países no desarrollados.

\section{REFERENCI AS BI BLI OGRÁFICAS}

1. Jiménez Paneque RE. Indicadores de calidad y eficiencia de los servicios hospitalarios: una mirada actual. Rev Cubana Salud Pública [Internet]. 2004[citado 13 Ago 2009]; 30(1):17-36. Disponible en:

http://scielo.sld.cu/scielo.php?script=sci_arttext\&pid=S0864-

34662004000100004\&lng=es\&nrm=iso\&ting=es

2. Jiménez R, Domínguez E, López L, Fariñas $H$. Difference between observed and predicted lenght of stay as indicator of patient care inefficiency. Internat J Quality Health Care. 1999; 11(5):375-84.

3. Martins $M$, Blais $R$, da Costa Leite I. Mortalidade hospitalar e tempo de permanência: comparação entre hospitais públicos e privados na região de Ribeirão Preto, São Paulo, Brasil. Cad Saúde Pública. 2004;20(Sup 2):268-82.

4. Omachonu VK, Suthummanon S, Akcin M, Asfour S. Predicting length of stay for Medicare patients at a teaching hospital. Health Serv Manage Res. 2004; 17(1): 1-12.

5. Tamargo Barbeito TO, Jiménez Paneque RE. El ajuste por riesgo en la evaluación del desempeño hospitalario. Rev Cubana Salud Pública [Internet]. 2009 [citado 13 Ago 2009]; 35(3). Disponible en:

http://bvs.sld.cu/revistas/spu/vol35_3_09/spu10309.htm 
6. Marazzi A, Gardiol L, Duong HD. New approaches to reimbursement schemes based on patient classification systems and their comparison. Health Serv Manage Res. 2007;20(3): 203-10.

7. Jiménez RE, Lam RM, Marot M, Delgado A. Observed-predicted length of stay for an acute psychiatric department, as an indicator of inpatient care inefficiencies. Retrospective case-series study. BMC Health Serv Res [Internet]. 2004 [citado 20 Marzo 2007]; 4(4). Disponible en: http://www.ncbi.nlm.nih.gov/pmc/articles/PMC387834/pdf/1472-6963-4-4.pdf

8. Liu Y, Phillips M, Codde J. Factors influencing patients' length of stay. Aust Health Rev. 2001;24(2):63-70.

9. Horn SD, Horn RA. Reliability and validity of the severity of illness index. Med Care. 1986; 24(2): 159-78.

10. Jiménez RE, Vázquez J, Fariñas $H$. Construcción y validación de un índice de gravedad para pacientes hospitalizados en áreas clínicas. Gaceta Sanitaria, Xunta de Galicia. 1997; 11: 122-30.

11. Abizanda C. Comparación de APACHE II y SAPS como indicadores pronósticos. Med Intens. 1990; 14(6): 259-63.

12. Librero J, Marín M, Peiró S, Verdaguer Manujos A. Exploring the impact of complications on length of stay in major surgery diagnosis-related groups. Internat J Quality Health Care. 2004;16:51-57.

13. Librero J, Peiró S, Ordiñana R. Comorbilidad crónica y homogeneidad de los grupos diagnósticos relacionados. Gaceta Sanitaria. 1999;13(4):292-302.

14. Myers RP, Quan H, Hubbard J N, Shaheen AA, Kaplan GG. Predicting in-hospital mortality in patients with cirrhosis: results differ across risk adjustment methods. Hepatology. 2009; 49(2):568-77.

15. Iucif N, Yazlle Rocha JS. Study of inequalities in hospital mortality using the Charlson comorbidity index. Rev Sáude Pública [Internet]. 2004 [citado $1 \mathrm{Abr}$ 2009]; 38(6). Available in: http://www.scielo.br/pdf/rsp/v38n6/en_05.pdf

16. Ramiarina RA, Ramiarina BL, Almeidall RM, Coelho de Albuquerque Pereira W. Comorbidity adjustment index for the International Classification of Diseases, 10th revision. Rev Saúde Pública. 2008;42(4):590-7.

17. Jiménez Paneque RE. Utilidad de la curva ROC. Nota Metodológica. FMC 2004; 11(8): 511-32.

18. Becchi MA, Belleli F, Clarizio M, Carulli N. Characteristics of patients admitted to a Division of Internal Medicine. Is hospital the best setting of care. Ann Ital Med Int. $2005 ; 20(4): 233-44$.

19. Everett G, Uddin N, Rudloff B. Comparison of Hospital Costs and Length of Stay for Community Internists, Hospitalists, and Academicians. J Gen Intern Med. $2007 ; 22(5): 662-7$. 
20. Horwitz Li, Kosiborod M, Lin Z, Krumholz HM. Changes in Outcomes for Internal Medicine Inpatients after Work-Hour Regulations. Ann Inter Med. 2007; 147(2): 97-103.

21. Sahavedan S, Earnest A, Koh $Y L$, Lee $\mathrm{KM}$, Soh $\mathrm{CH}$, Ding YY. Improving the diagnosis related grouping model's ability to explain length of stay of elderly medical inpatients by incorporating function-linked variables. Ann Acad Med Singapore. 2004;33(5):614-22.

22. Gursel G, Demirtas S. Value of APACHE II, SOFA and CPIS Scores in Predicting Prognosis in Patients with Ventilator-Associated Pneumonia. Respiration. 2006; 73:503-8.

23. Hartz AJ, Bade PF, Sigmann P, Guse C, Epple P, Goldberg KC. The evaluation of screeening methods to identify medically unnecessary hospital stay for patient with pneumonia. Int J. 1996; 8: 3-11.

24. Bernard AM, Mynard R, Rosevear JS, McMahon LF. The integrated inpatient management. Med Care. 1995; 7:663-75.

25. Ryan S, Lowe CS, Hardes G. A quantitative approach to quality inprovement and resource allocation. Qual Clin Pra. 1995; 15:11-6.

* Variable de diseño o variable ficticia, es un recurso de la estadística para introducir en los modelos matemáicos las variables que no son un número y poder trbajar con ellas.

Recibido: 10 de agosto de 2011.

Aprobado: 13 de septiembre de 2011.

Teddy Osmin Tamargo Barbeito. Hospital Clinicoquirúrgico "Hermanos Ameijeiras". San Lázaro No. 701 esq. a Belascoaín. Centro Habana 10300. La Habana, Cuba. Correo electrónico: teosmin@infomed.sld.cu 